\title{
Monitoring the Electrochemical Energy Storage Processes of an Organic Full Rechargeable Battery via Operando Raman Spectroscopy - A Mechanistic Study
}

\author{
Xiu-Mei Lin, ${ }^{*},+$, De-Yin Wu, ${ }^{,} \S$ Ping Gao, ${ }^{\dagger}$ Zhi Chen," Mario Ruben," Maximilian Fichtner ${ }^{*}, \dagger, \|$
}

${ }^{\dagger}$ Helmholtz Institute Ulm for Electrochemical Energy Storage (HIU), Helmholtzstr. 11, 89081 Ulm, Germany

\#Department of Physics, Xiamen University, 361005 Xiamen, China

§State Key Laboratory of Physical Chemistry of Solid Surfaces, iChEM, College of Chemistry and Chemical Engineering, Xiamen University, 361005 Xiamen, China

"Institute of Nanotechnology, Karlsruhe Institute of Technology (KIT), P.O. Box 3640, 76021 Karlsruhe, Germany

*E-mail: xiu-mei.lin@xmu.edu.cn

dywu@xmu.edu.cn

m.fichtner@kit.edu

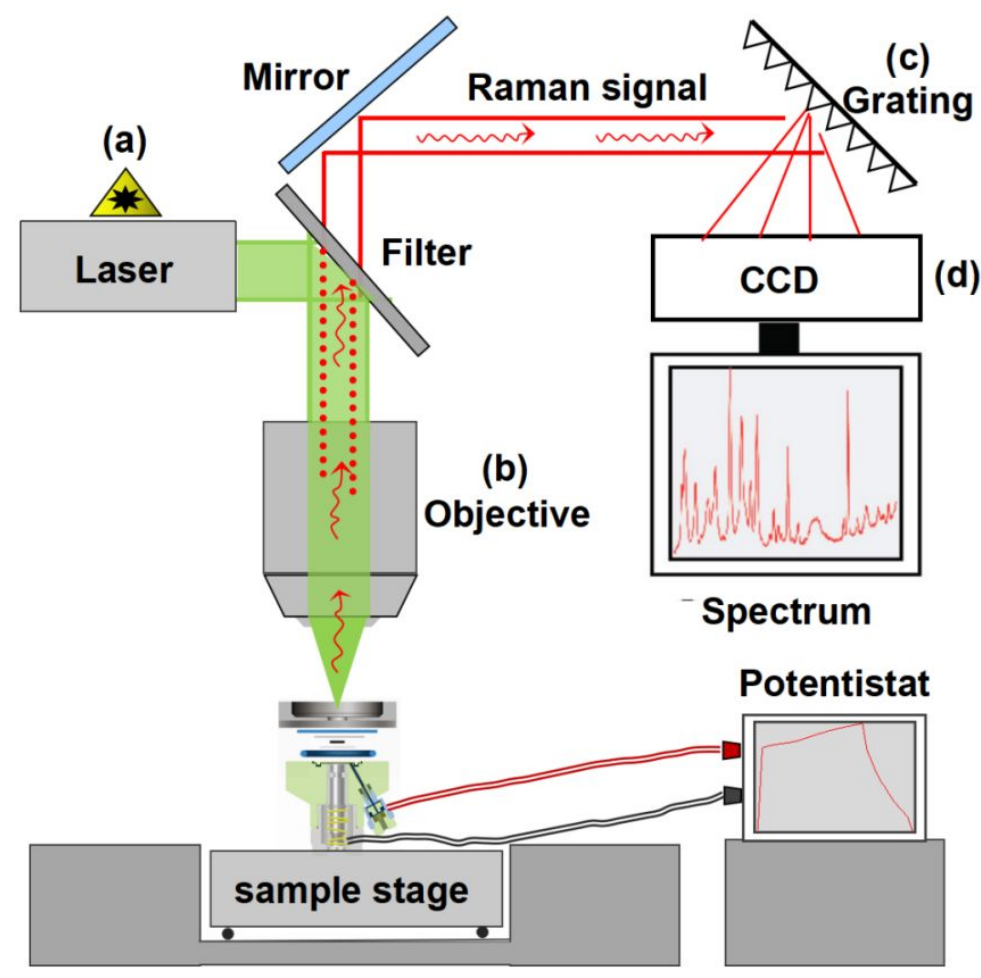

Figure. S1. Schematic illustration of the operando electrochemical Raman spectroscopy system. 


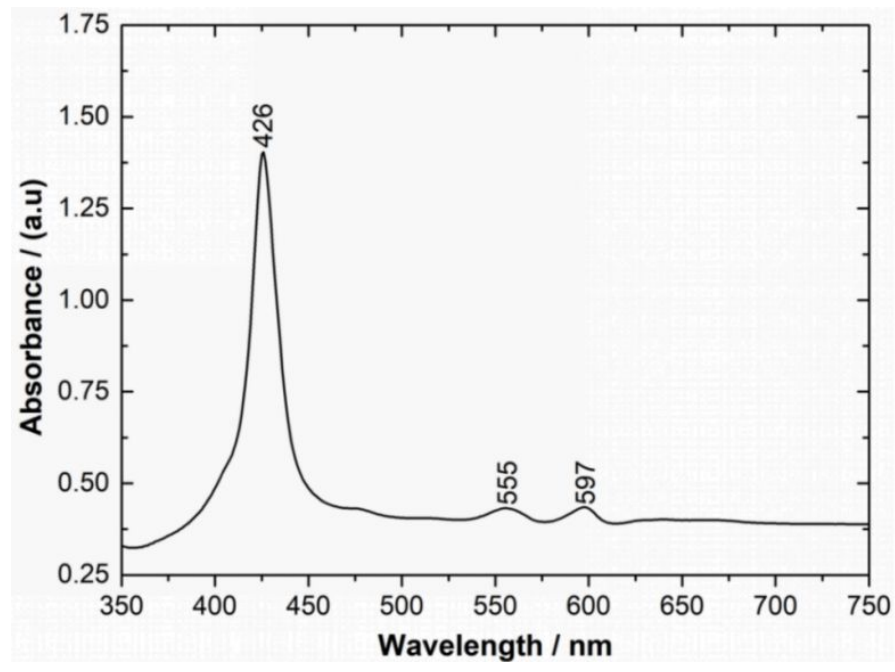

Figure. S2. UV-Vis adsorption spectrum of CuDEPP.

\section{Calculation of theoretical capacity of CuDEPP:}

Formula of CuDEPP: $\mathrm{C}_{36} \mathrm{H}_{20} \mathrm{CuN}_{4}$; Formula weight of CuDEPP: $572.14 \mathrm{~g} / \mathrm{mol}$

Theoretical capacity=nF/M=2*96485*1000/(572.14*3600)=93.69 mA h g ${ }^{-1}$

n: the number of transfer electrons; F: Faraday constant (96485 C); M: Formula weight 


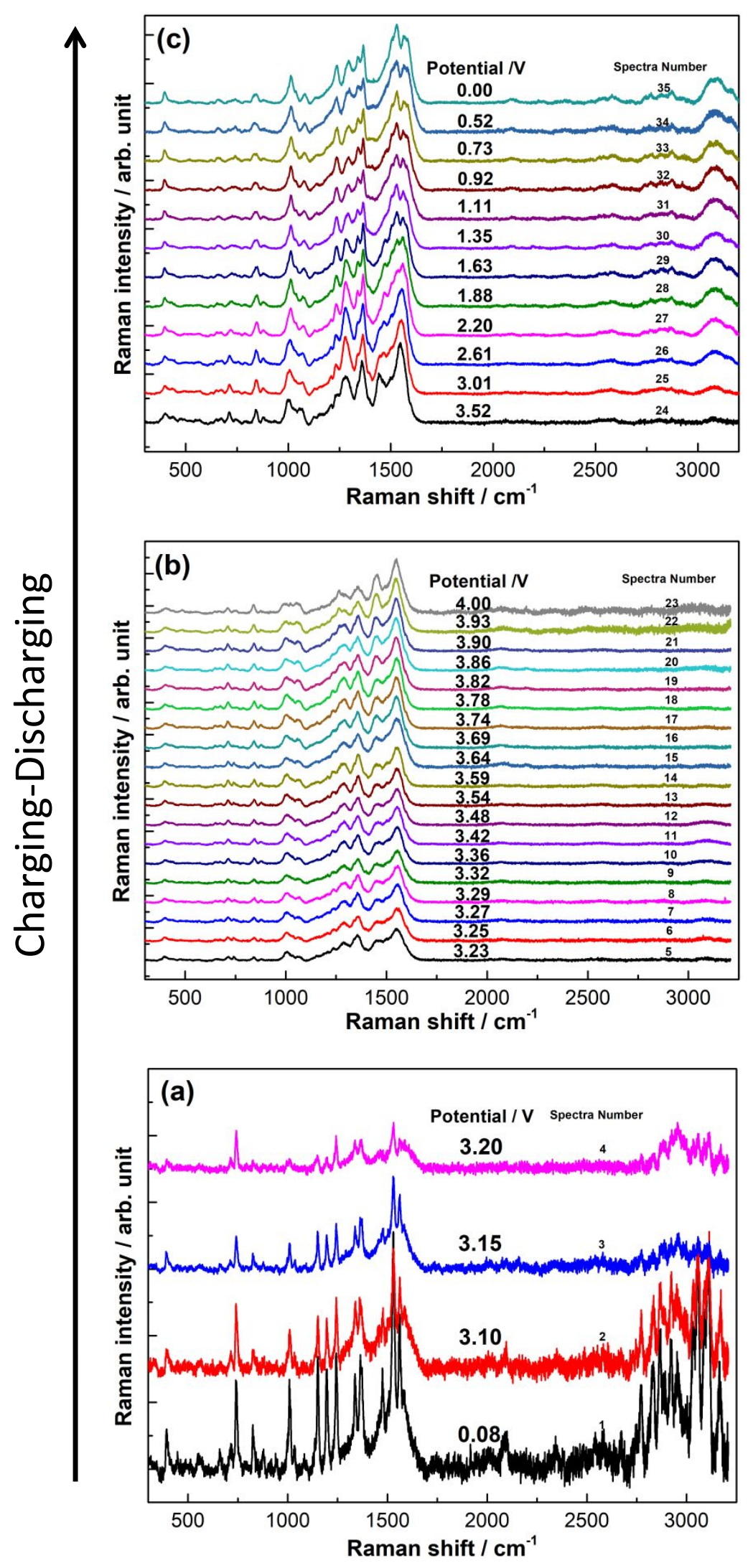

Figure. S3. (a)-(c). Series of Raman spectra of the CuDEPP anode during initial charge-discharge cycling with a full spectral range from $300 \mathrm{~cm}^{-1}$ to $3200 \mathrm{~cm}^{-1}$ and with the corresponding potential on each spectrum. 

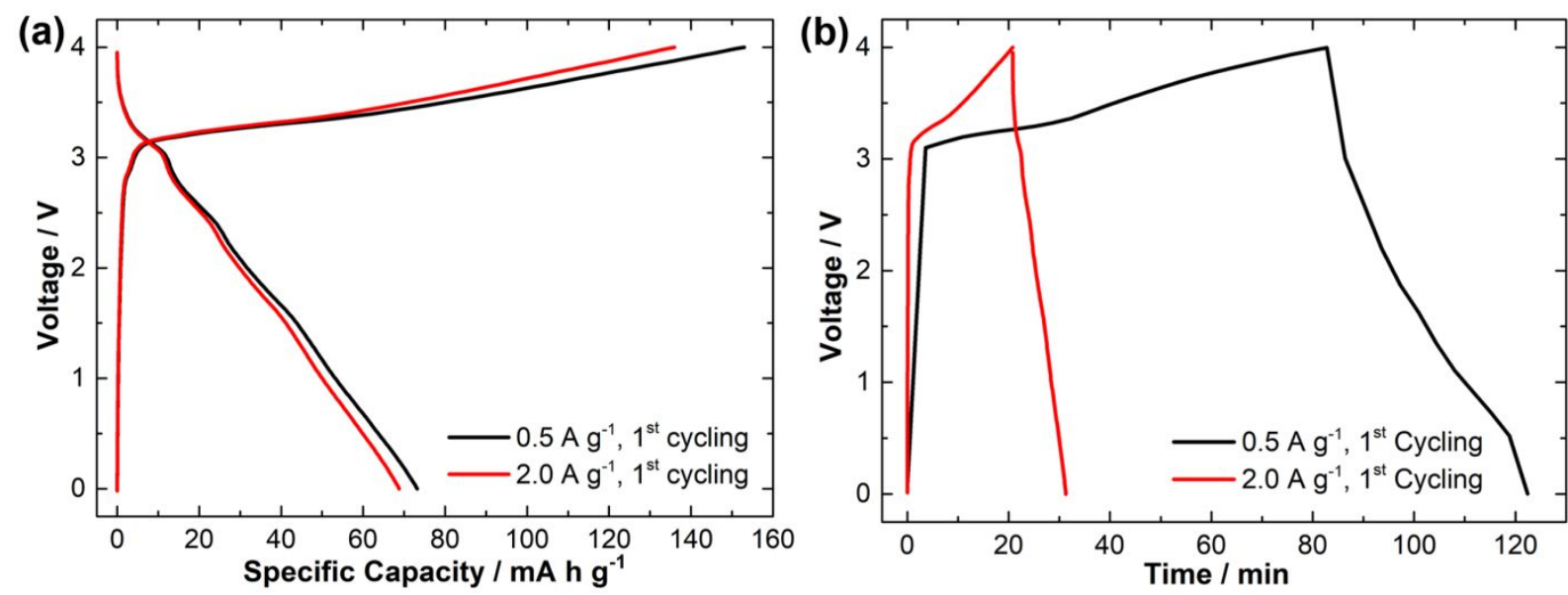

Figure. S4. (a) Galvanostatic charge-discharge profiles; (b) "Voltage vs cycling time" curves of the CuDEPP / PP14TFSI / Graphite organic full rechargeable battery for the $1^{\text {st }}$ cycle at specific current of $0.5 \mathrm{~A} \mathrm{~g}^{-1}$ and $2.0 \mathrm{~A} \mathrm{~g}^{-1}$ respectively.

Table S1: Band assignment of the Raman spectra of CuDEPP ${ }^{1-7}$

\begin{tabular}{|c|c|c|}
\hline \multicolumn{2}{|l|}{ Frequency $/ \mathrm{cm}^{-1}$} & \multirow[b]{2}{*}{ Assignmen } \\
\hline Observed & References & \\
\hline $\begin{array}{l}3164,3110,3089,3057,3035,2957, \\
2925,2865,2833,2769\end{array}$ & $2700-3200$ & $v \mathrm{CH}$ \\
\hline $2090,2070,2090$ & $2000-2200$ & $v \mathrm{C} \equiv \mathrm{C}$ \\
\hline $1557,1525,1546,1557,1525$ & 1564,1531 & $v \mathrm{C}_{\mathrm{Pr}} \mathrm{C}_{\mathrm{m}}$ \\
\hline $1471,1447,1471$ & 1477 & $v \mathrm{C}_{\mathrm{Pr}} \mathrm{C}_{\mathrm{Pr}}$ \\
\hline $1371,1337,1356,1371,1337$ & 1372,1340 & $v \mathrm{C}_{\mathrm{Pr}} \mathrm{C}_{\mathrm{Pr}}$ \\
\hline 1287 & 1290 & $v \mathrm{C}_{\mathrm{Pr}} \mathrm{N}$ \\
\hline 1244 & 1241 & $v \mathrm{C}_{\mathrm{Ph}} \mathrm{C}_{\mathrm{m}}$ \\
\hline $1081,1062,1081$ & 1080 & $\delta \mathrm{C}_{\mathrm{Pr}} \mathrm{H}$ \\
\hline 1010 & 1006 & $\delta \mathrm{C}_{\mathrm{Ph}} \mathrm{C}_{\mathrm{Ph}} \mathrm{C}_{\mathrm{Ph}}$ \\
\hline 876 & 884 & $\delta \mathrm{C}_{\mathrm{Ph}} \mathrm{C}_{\mathrm{Ph}} \mathrm{C}_{\mathrm{Ph}}$ \\
\hline 824,845 & 849 & $\delta \mathrm{C}_{\mathrm{Pr}} \mathrm{C}_{\mathrm{m}} \mathrm{C}_{\mathrm{Pr}}$ \\
\hline 739 & 742 & $\delta \mathrm{C}_{\mathrm{Pr}} \mathrm{C}_{\mathrm{Pr}} \mathrm{C}_{\mathrm{Pr}}$ \\
\hline 712 & 696 & $\delta \mathrm{C}_{\mathrm{Ph}} \mathrm{C}_{\mathrm{Ph}} \mathrm{C}_{\mathrm{Ph}}$ \\
\hline 395 & 410 & $v \mathrm{CuN}$ \\
\hline
\end{tabular}

$\mathrm{C}_{\mathrm{Pr}}$ : pyrrole carbons; $\mathrm{C}_{\mathrm{Ph}}$ : phenyl carbons; $\mathrm{C}_{\mathrm{m}}$ : methine bridge carbons; $v$ : stretching; $\delta$ : in-plane deformation. 


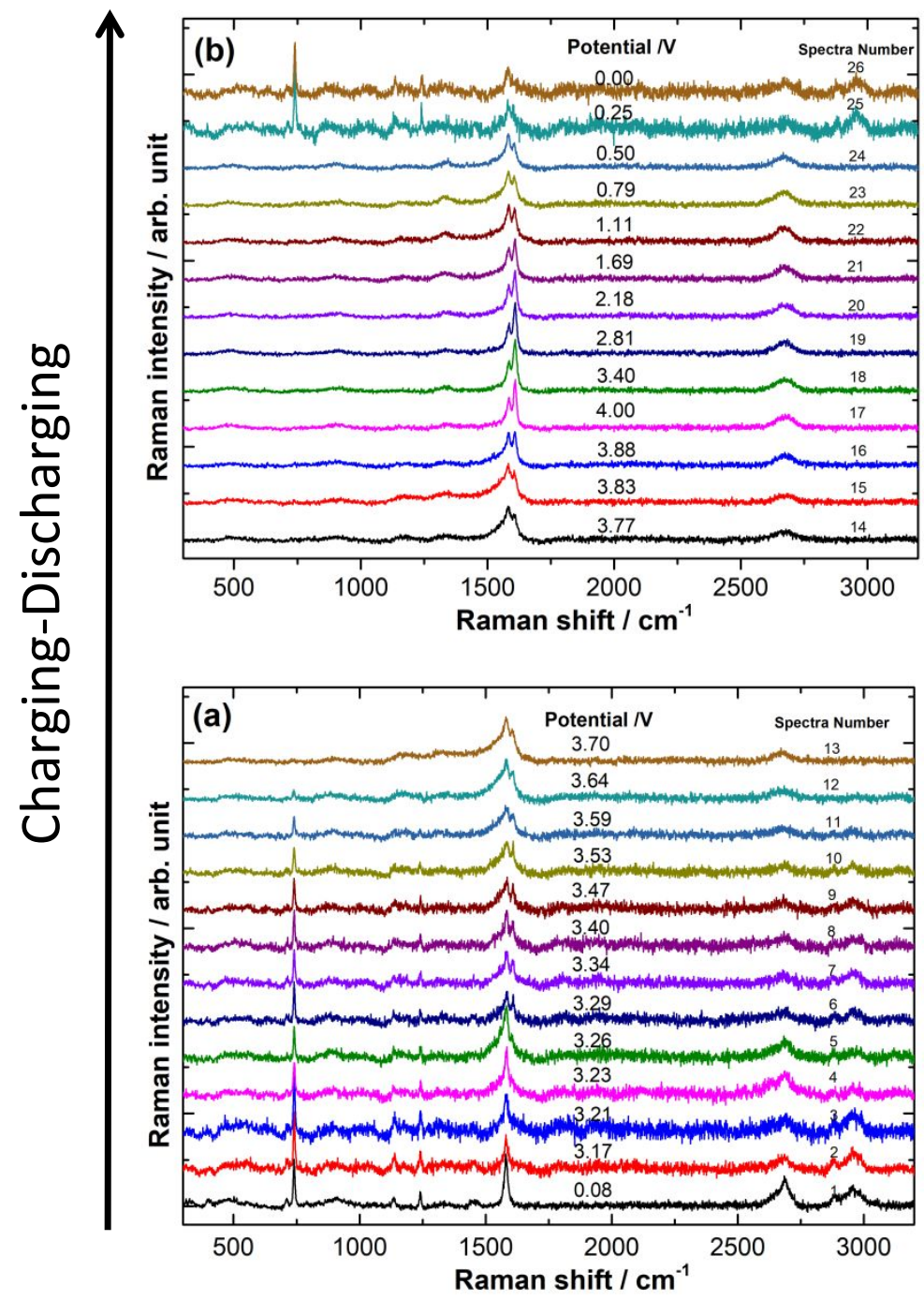

Figure S5. (a), (b). Series of Raman spectra of the graphite cathode during initial charge-discharge cycling with a full spectral range from $300 \mathrm{~cm}^{-1}$ to $3200 \mathrm{~cm}^{-1}$ and with the corresponding potential on each spectrum. 


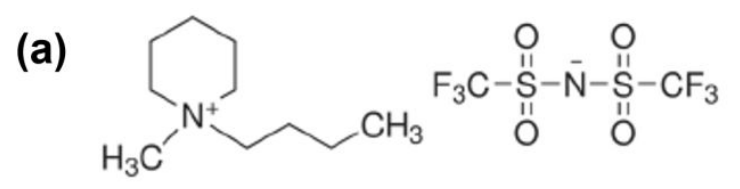

(b)

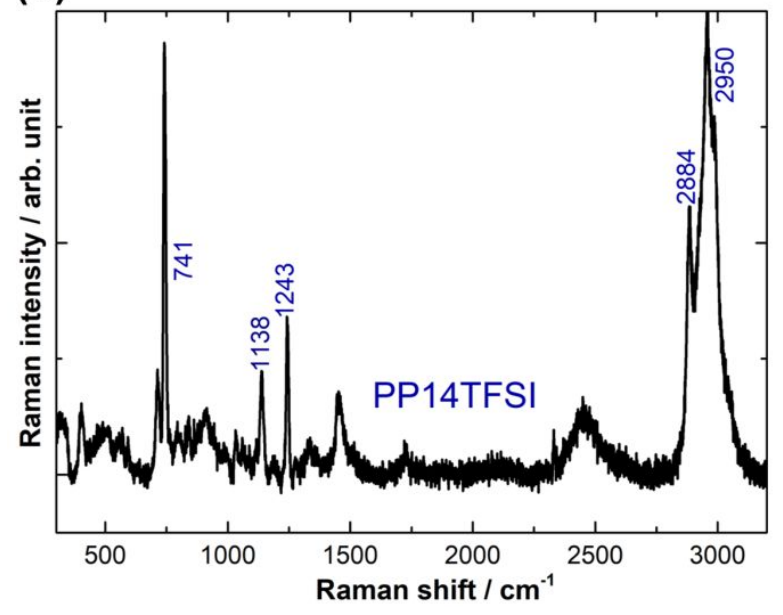

Figure S6. Molecular structure (a) and Raman spectrum (b) of PP14TFSI ionic liquid electrolyte.

\section{REFERENCES}

(1) Liao M.-S.; Scheinera, S. Electronic structure and bonding in metal porphyrins, metal=Fe, Co, Ni, Cu, Zn. J. Chem. Phys. 2002, 117 (1), 205219.

(2) Sengupta, K.; Chatterjee, S.; Samanta, S.; Dey, A. Direct observation of intermediates formed during steady-state electrocatalytic $\mathrm{O}_{2}$ reduction by iron porphyrins. Proc. Natl. Acad. Sci. USA 2013, 110 (21), 8431-8436.

(3) Silva, C. D.; Czarnecki, K.; Ryan, M. D. Visible and resonance Raman spectra of low valent iron Porphy. Inorganica Chimica Acta 1999, 287 (1), 21-26.

(4) Atamian, M.; Donohoe, R. J.; Lindsey, I. J. S.; Bocian, D. F. Resonance Raman Spectra and Normal-Coordinate Analysis of Reduced Porphyrins. 1. Zinc(II) Tetraphenylporphyrin Anion. J. Phys. Chem. 1989, 93 (6), 2236-2243.

(5) LeCours, S. M.; Philips, C. M.; Paula, J. C.; Therien, M. J. Synthesis, Transient Absorption, and Transient Resonance Raman Spectroscopy of Novel Electron Donor-Acceptor Complexes: [5,15-Bis[(4'-nitrophenyl)ethynyl]-10,20-diphenylporphinato]copper(II) and [5-[[4'(Dimethylamino)phenyl] ethynyl]-15-[(4'nitrophenyl)ethynyl]-10,20-diphenylporphinato]copper(II). J. Am. Chem. Soc. 1997, 119 (51), 1257812589.

(6) Terner, J.; Palaniappan, V.; Gold, A.; Weiss, R.; Fitzgerald, M. M.; Sullivan, A. M.; Hosten, C. M. Resonance Raman spectroscopy of oxoiron(IV) porphyrin p-cation radical and oxoiron(IV) hemes in peroxidase intermediates. J. Inorg. Biochem. 2006, 100 (4), $480-501$.

(7) Teraoka, J.; Hashimoto, S.; Sugimoto, H.; Mori, M.; Kitagawa, T. Resonance Raman Characterization of Highly Reduced Iron Octaethylporphyrin: [Fe(OEP)]"' (n=0, 1, and 2). J. Am. Chem. Soc. 1987, 109 (1), 180-184. 